\title{
Hierarchical triple star systems towards the Galactic Bulge through OGLE's eye
}

\author{
T. Hajdu ${ }^{1}$, T. Borkovits ${ }^{1,2}$, E. Forgács-Dajka ${ }^{3}$, J.Sztakovics ${ }^{3,4}$, G. \\ Marschalkó $^{3}$ and G. Kutrovátz ${ }^{5}$ \\ 1 Konkoly Observatory, Research Centre for Astronomy and Earth Sciences, \\ H-1121 Budapest, Konkoly Thege Miklós út 15-17, Hungary (E-mail: \\ t.hajdu@astro.elte.hu) \\ 2 Baja Astronomical Observatory of Szeged University, H-6500 Baja, Szegedi \\ ut, Kt. 766, Hungary \\ 3 Eötvös Loránd University, Department of Astronomy, Hungary \\ ${ }^{4}$ Eszterházy Károly University, Department of Physics, Hungary \\ 5 Budapest University of Technology and Economics, Department of \\ Philosophy and History of Science, Hungary
}

Received: October 31, 2019; Accepted: January 3, 2020

\begin{abstract}
We report the result of eclipse timing variation (ETV) analyses of OGLE-IV eclipsing binaries (EBs). We have identified around 1000 potential triple (or multiple) system candidates and, in addition, we determined the orbital parameters and we carried out statistical analyses of the properties of the candidates. We found that (i) the distribution of the outer eccentricity has a maximum around $e_{2}=0.3$; (ii) in most cases the estimated outer mass ratio is lower than $q_{2} \sim 0.5$. Besides, we also present some systems that deserve special attention.
\end{abstract}

Key words: binaries: close - binaries: eclipsing - methods: numerical

\section{Introduction}

The investigation of triple stellar systems is not only important to understand the orbital evolution of the close binaries, but also to study their life (Toonen et al., 2016). Furthermore, the various formation theories of close binary systems, e. g. the so called Kozai Cycles with Tidal Friction (KCTF) mechanism (see, e. g. Kiseleva et al., 1998; Fabrycky \& Tremaine, 2007; Naoz \& Fabrycky, 2014), as well as the recently proposed different disk and core fragmentation processes (Tokovinin, 2018; Moe \& Kratter, 2018), require the presence of a third component to explain the large number of non-evolved binary systems with very short orbital period.

The Optical Gravitational Lensing Experiment (OGLE) was designed for discovering dark matter using the microlensing technique in 1992 (Udalski et al., 
1992). Recent and past OGLE surveys were found to be useful e.g. for exoplanet exploration (Bouchy et al., 2004) and for the investigation of variable stars (Soszyński et al., 2016). The Galactic Bulge part of the OGLE-IV survey with its approximately $450000 \mathrm{EBs}$, which were identified by Soszyński et al. (2016) gives us a good chance to increase the number of the candidates of hierarchical triple stellar systems.

In this paper we present the results of searching for hierarchical triple star candidates towards the Galactic Bulge with ETV analyses of EBs observed during the $O G L E-I V$ survey.

\section{Third body effects on the ETV}

One of the best known methods for the identification of a third companion around an eclipsing binary is based on the detection and analysis of the eclipse timing variations of the binary. If an EB has a distant, third companion, its distance from the observer varies periodically due to the EB's revolution around the common center of mass of the system. As a natural consequence, the lighttravel time effect (LTTE) occurs.

If the third component significantly approaches the EB then other effects called dynamical perturbations also appear which have a very complicated dependence on the orbital elements. The most thorough discussion of these effects can be found in Borkovits et al. (2015).

\section{Data processing}

The OGLE-IV survey provided around 425000 light curves of EBs. Unfortunately most of them do not contain enough data points for a detailed examination. Therefore we investigated only those systems whose light curves contain more than 4000 points. Applying this criterion, we reduced our sample to $\sim 80000$ systems.

To generate and analyse $\mathrm{O}-\mathrm{C}$ (Observed minus Calculated) diagrams we used an automatic algorithm. It needs only the orbital period of the EB and its light curve (LC) as input. It uses the folded and binned LC of an EB to determine the phase borders of the eclipses and to generate template LCs for them.

To get more precise $\mathrm{O}-\mathrm{C}$ curves for the ETV analyses we used several consecutive eclipses to determine only one point since mostly the individual cycle to cycle LCs are poorly covered. The program fits the parameters of the primary and the secondary minima at once, therefore we get a kind of averaged $\mathrm{O}-\mathrm{C}$ diagram. As a next step, a combined grid-search and Levenberg-Marquardt algorithm was used to search for candidates and determine the parameters of the LTTE. More details about this method and the results can be found in Hajdu et al. (2019). 
The above method is effective for searching systems with long tertiary periods. However, there are several systems which have relatively short periods and their ETV shows significant dynamical effects. These types of systems can be found if we determine all possible times of eclipse minima. This can be done in our case, if the LC is poorly covered, if we fit only the shifting parameter which represents the $\mathrm{O}-\mathrm{C}$ point.

\section{Results}

In conclusion, we have found about a thousand hierarchical stellar system candidates with the first method. Four of them are possible quadruple systems based on their residual ETVs. A third component is a possible brown dwarf based on the its mass function and the mass of the W UMa close binary (Dimitrov \& Kjurkchieva, 2015). We have also found several new triples with relatively short outer period $\left(P_{2}<300^{d}\right)$ using the second method.

\subsection{Statistical analyses of the parameters}

Due to the large number of triple system candidates, it is worth while to examine distributions of the parameters determined with the LTTE delays.

Owing to the fact that the derived eccentricities have a large uncertainty, we used the Kernel Density Estimation method to determine the dispersion of the outer eccentricities. The distribution has a significant peak around $e_{2} \approx 0.3$

Figure 1 represents the period-period correlation of the candidates which shows a grouping around outer period $P_{2} \approx 2000^{d}$ which equals the length of the observation. It is possible that if the real outer period is significantly longer than our data length, then the LM-fit more likely converges to a lower period value that is closer to the duration of the time span.

In the absence of the known binary masses in our sample, the minimum masses of the tertiary components were estimated from the mass function with the assumption that $m_{A B} \simeq 2 M_{\odot}$. Regarding our sample, one finds that the vast majority of the candidate systems have minimum outer masses less than $1 M_{\odot}$, i.e. an outer mass ratio of $q_{2 \min }<0.5$.

Acknowledgements. This project has been partly supported by the Development and Innovation Office, NKFIH-OTKA grants K-115709 and KH-130372, and the Lendület Program of the Hungarian Academy of Sciences, project No. LP2018-7/2018.

\section{References}

Borkovits, T., Rappaport, S., Hajdu, T., \& Sztakovics, J., Eclipse timing variation analyses of eccentric binaries with close tertiaries in the Kepler field. 2015, Mon. Not. R. Astron. Soc., 448, 946, DOI: 10.1093/mnras/stv015 


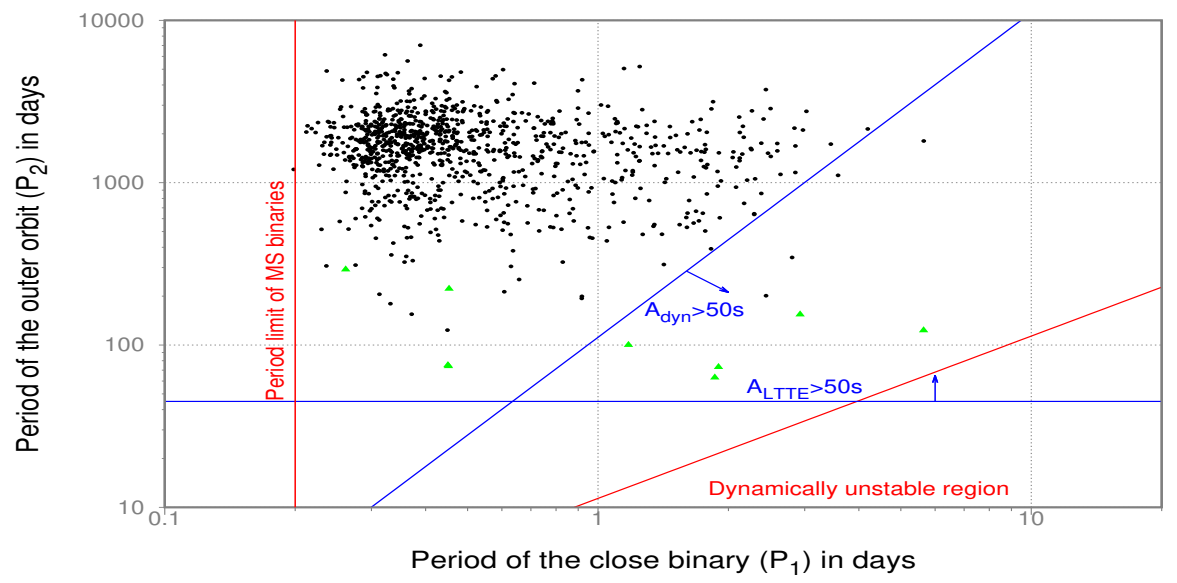

Figure 1. The location of the triple star candidates based on the first method (black dots) and based on the second method (green triangles) in the $P_{1}$ vs $P_{2}$ plane.

Bouchy, F., Pont, F., Santos, N. C., et al., Two new "very hot Jupiters" among the OGLE transiting candidates. 2004, Astron. Astrophys., 421, L13, DOI: 10.1051/0004-6361:20040170

Dimitrov, D. P. \& Kjurkchieva, D. P., Ultrashort-period main-sequence eclipsing systems: new observations and light-curve solutions of six NSVS binaries. 2015, Mon. Not. R. Astron. Soc., 448, 2890, DOI: 10.1093/mnras/stv147

Fabrycky, D. \& Tremaine, S., Shrinking Binary and Planetary Orbits by Kozai Cycles with Tidal Friction. 2007, Astrophys. J., 669, 1298, DOI: 10.1086/521702

Hajdu, T., Borkovits, T., Forgács-Dajka, E., et al., Eclipse timing variation analysis of OGLE-IV eclipsing binaries towards the Galactic Bulge - I. Hierarchical triple system candidates. 2019, Mon. Not. R. Astron. Soc., 485, 2562, DOI: 10.1093/mnras/stz592

Kiseleva, L. G., Eggleton, P. P., \& Mikkola, S., Tidal friction in triple stars. 1998, Mon. Not. R. Astron. Soc., 300, 292, DOI: 10.1046/j.1365-8711.1998.01903.x

Moe, M. \& Kratter, K. M., Dynamical Formation of Close Binaries during the Premain-sequence Phase. 2018, Astrophys. J., 854, 44, DOI: 10.3847/1538-4357/aaa6d2

Naoz, S. \& Fabrycky, D. C., Mergers and Obliquities in Stellar Triples. 2014, Astrophys. J., 793, 137, DOI: 10.1088/0004-637X/793/2/137

Soszyński, I., Pawlak, M., Pietrukowicz, P., et al., The OGLE Collection of Variable Stars. Over 450000 Eclipsing and Ellipsoidal Binary Systems Toward the Galactic Bulge. 2016, Acta Astron., 66, 405

Tokovinin, A., Dancing Twins: Stellar Hierarchies That Formed Sequentially? 2018, Astron. J., 155, 160, DOI: 10.3847/1538-3881/aab102 
Toonen, S., Hamers, A., \& Portegies Zwart, S., The evolution of hierarchical triple star-systems. 2016, Computational Astrophysics and Cosmology, 3, 6, DOI: 10.1186/s40668-016-0019-0

Udalski, A., Szymanski, M., Kaluzny, J., Kubiak, M., \& Mateo, M., The Optical Gravitational Lensing Experiment. 1992, Acta Astron., 42, 253 